\title{
LA REPETICIÓN EN EL DISCURSO DEL PROFESOR DE ELE: FORMAS Y FUNCIONES
}

\author{
M. ${ }^{a}$ Vicenta González Argüello \\ Universidad de Barcelona \\ Grupo DRE_DIX \\ vicentagonzalez@ub.edu
}

\begin{abstract}
Resumen
La repetición es un mecanismo discursivo característico de la lengua oral. Este trabajo se plantea abordar dicho mecanismo en el discurso del profesor de español como lengua extranjera, para ello se describen sus características formales y se analizan sus funciones comunicativas con el fin de establecer en qué casos llega a ser efectiva. Todo ello teniendo en cuenta los trabajos existentes sobre la repetición en el discurso didáctico y en la lengua conversacional. El corpus que se ha analizado está formado por grabaciones de 10 sesiones de clase de 10 profesores de español diferentes.
\end{abstract}

PALABRAS CLAVE: discurso didáctico; discurso del profesor, español lengua extranjera; repetición.

\begin{abstract}
Repetition is a discursive mechanism that characterises the oral language. This work plans to approach this mechanism in the discourse of the Spanish as a Foreign Language teacher. And for that purpose, its formal characteristics are described, and its communicative functions are analysed -ultimately aiming to establish the cases in which it is effective. Existing works about repetition in the didactic discourse and in the conversational language are taken into account. The corpus analysed here consists of recordings of 10 teaching sessions by 10 different Spanish teachers.

KEY WORDS: Didactic discourse, Teacher's discourse, Spanish as a Foreign Language; Repetition
\end{abstract}

\section{Introducción}

Las interacciones entre hablantes nativos y no nativos se caracterizan por el despliegue de diferentes estrategias por parte de los interlocutores para evitar los bloqueos comunicativos y facilitar así la comunicación. Entre estas estrategias se incluye la repetición de los enunciados emitidos; por ello no ha de extrañar que esta sea también una estrategia utilizada por el profesor en su discurso en interacción con sus alumnos en el aula de lenguas extranjeras. La repetición es una de las características propias del Foreigner Talk o habla dirigida a extranjeros (Ferguson, 1977; Hatch, 1992; Stern, 1983; Lightbown y Spada, 1993, entre otros), y del Teacher Talk o discurso del profesor (Chaudrom, 1983, 1988; Allwright, 1988; Salaberri, 1988; entre otros). Entre los estudios que 
han analizado la repetición en el discurso didáctico del profesor de español como lengua extranjera (a partir de ahora ELE) destacan el de Aymerich y Díaz (1992). Pero también la repetición es una estrategia discursiva constante en el habla entre nativos, es necesario, pues, si se quiere establecer cuáles son las funciones de esta estrategia en el discurso generado en el aula de lenguas partir de los estudios de la conversación para poder establecer si las funciones que cumple en una y en otra situación comunicativa son equiparables. Todo ello con el fin último de establecer la efectividad del discurso didáctico (Mercer, 2009).

\section{Objetivos del trabajo}

El estudio que se presenta a continuación se basa en el análisis de 10 sesiones de enseñanza-aprendizaje grabadas a diez profesores diferentes destinadas a alumnos de nivel principiante; $y$ tiene como objetivo establecer cuáles son las funciones que cumple la repetición en el discurso del profesor de español como lengua extranjera. Para ello se parte de los estudios que analizan esta estrategia discursiva en el ámbito de la conversación espontánea con el fin de contrastar si cumple funciones similares.

El trabajo se inicia con una revisión de los estudios de la conversación centrados en la repetición, para a continuación presentar las diferentes tipos de repetición que se han dado en los discursos analizados; seguidamente, se enumeran las funciones de estas repeticiones en los discursos generados por el profesor en el aula; a continuación se analiza si cumplen los objetivos que han propiciado su aparición; para terminar comparando las funciones de la repetición en el discurso didáctico con las que esta presenta en la conversación.

\section{La repetición en la conversación}

Una de las características de la conversación que ha sido objeto de estudio desde mediados del siglo pasado es la repetición (Martinell, 1974; Tannen, 1989; Vigara, 1992, 1995; Herrero, 1995; Briz, 1998a, 1998b). Estos autores tratan este fenómeno contemplado desde diferentes puntos de vista, ya sea como un recurso de cohesión como afirma Briz (1998) ${ }^{1}$, ya sea como una característica intrínseca a la lengua, pues parece imposible emitir enunciados que nunca antes hayan sido producidos, como afirma Tannen D. (1989).

Es de gran interés para nuestra investigación la idea que Tannen desarrolla en su obra (Repetition in conversation: toward a poetics of talk, 1989).

1 La idea de repetición como mecanismo de cohesión, no es una aportación nueva. Hay autores que hace ya varias décadas que así lo consideran como Hallyday y Hasan (1967), o E. Martinell (1974). 
Analysis of repetition thus sheds light on our conception of language production, or, as Becker would say, 'languaging'. In shot, it suggests that language is less freely generated, more prepatterned, than most current linguistic theory acknowledges. This is not however, to say that speakers are automatons, cranking out language by rote. Rather, propatterning (or idiomaticity, or formulacity) is a resource for creativity. It is the play between fixity and novelty that makes possible the creation of meaning. (Tannen, 1989:37)

Tal gradación va desde las expresiones a modo de estructuras lexicalizadas dentro del código que se establece entre los interlocutores, y cuya omisión sería notada y desaprobada por estos, hasta otras expresiones más libres que se van configurando y llenando de valor dentro de la interacción en la que surgen, como algunas repeticiones totales o las repeticiones eco. La autora apunta que para poder detectar el grado de fijeza de algunas expresiones, y, por lo tanto, percibir que son repeticiones de otras producciones ya existentes hay que contemplar las tres dimensiones que intervienen en la producción de la lengua y que son las que determinan el grado de fijación: la forma, el contexto y el tiempo. Es interesante relacionar la información que nos aporta con lo que sucede en el aula en situación de enseñanza, son muchos los enunciados que a modo de ritual ya interiorizado por todos los participantes se repiten en cada sesión de clase; por ejemplo a la hora de presentar un nuevo tema, de introducir acciones tales como la realización de actividades o la revisión de ejercicios, etc.

Esta autora clasifica los diferentes tipos de repetición que pueden darse en la conversación a partir de su función; así Tannen (1989) presenta 4 funciones²:

2 Vigara (1995: 180) presenta una clasificación similar a la de Tannen al establecer las funciones de la repetición en la conversación:

"a) proporciona a la nueva información un "marco de referencia" estable en el que encauzarse e integrarse;

b) contribuye a la fluidez emisora del hablante, que puede encontrar en lo previamente formulado parte de la nueva emisión ya lista;

c) facilita la comprensión del oyente, proveyéndole información aproximadamente al mismo ritmo y en la misma proporción que el hablante la produce (piénsese, en cambio, en un texto leído, que obliga a decodificar y asimilar la información a un ritmo mucho más rápido que el que el autor ha empleado en elaborarla);

d) evidencia la actitud del hablante, el modo en que se asume su relación interlocutiva y contribuye al sentido del discurso;

e) actúa como un dispositivo de cohesión más (Halliday y Hasan, 1976), que muestra cómo las nuevas emisiones están unidas al discurso propio y cómo las ideas presentadas en el discurso están unidas unas a otras;

f) cumple fines sociales de interacción, en la medida en que vincula a los hablantes regula el flujo discursivo y administra la tarea de la interpretación durante la conversación (cf. Tannen , 1989)." 
a) permitir emitir producciones que cumplan la función de evitar los silencios;

b) facilitar la comprensión de los enunciados al interlocutor;

c) conectar los diferentes enunciados entre sí;

d) facilitar la interacción; y

e) dotar de coherencia a la conversación a través de la implicación interpersonal.

Además, esta estrategia puede clasificarse atendiendo a su forma (Tannen, 1989):

a) autorrepetición, que es la repetición de lo emitido por uno mismo;

b) alo-repetición, cuando se repite lo emitido por otros;

c) repetición exacta;

d) repetición con variaciones, si presenta cambios en cuanto a la entonación, o si varían algunas palabras o frases, si hay cambios de persona gramatical o tiempo verbal; y

e) los patrones rítmicos, en los que palabras totalmente diferentes se estructuran de igual forma sintáctica y rítmicamente que la estructura que le precede.

También Briz (1998a: 73, 1998b: 37) apunta que es posible diferenciar dos tipos de repetición "[...] la monológica, la que se da en la intervención de un hablante, o dialógica, es decir, aquella que aparece en intervenciones de distintos hablantes." Este autor, al tratar la repetición, aborda, además, la reelaboración. Ya que parece que esta estrategia es una manifestación de la repetición que incluye algún tipo de transformación. Parece que la reelaboración obedece a una necesidad del hablante de aclarar el enunciado emitido.

Así, pues, parece que el concepto de repetición es bastante amplio, y que puede incluir también las reformulaciones, como una manifestación más de la repetición.

Otro tipo de repetición que ha sido objeto de estudio por Vigara (1995) son los ecos retrospectivos o de progresión ilocutiva, en los que el hablante repite literalmente lo dicho por su interlocutor, incorporándolo a su acto de habla y al servicio de su particular intención de comunicación, con una referencia y un sentido nuevos. Sobre este tipo de repetición hay que mencionar a G. Herrero (1995)3 a partir del estudio de la conversación, en su trabajo pro-

3 Sobre las construcciones eco, también hay que mencionar el trabajo de D. Dumitrescu (1993): Función pragma-discursiva de la interrogación ecoica usada como respuesta en español. Este, a partir del análisis de este tipo de construcciones, presenta una clasificación de las interrogaciones ecoicas según la función que desempeñan en la conversación. 
fundiza en un tipo de repetición muy específica: las construcciones-eco, consideradas como "[...] construcciones que repiten de forma exacta o con ligerísimos cambios que reflejan las nuevas condiciones de la enunciación [...] enunciados previos o parte de los mismos, producidos en el turno de habla inmediatamente anterior al turno en que ellas se realizan." (Herrero, 1995: 126)

La idea de Tanen (1989:37), ya apuntada, ("It is the play between fixity and novelty that makes possible the creation of meaning") podría estar en la base del uso, mayoritariamente inconsciente, que los profesores hacen de la repetición en su discurso en la situación de enseñanza-aprendizaje. Aunque, como ella misma afirma, la repetición ha de entenderse como un fenómeno que se da en diferentes grados dentro de la interacción que se produce en las interacciones conversacionales, quizá esta variación ha de compararse con lo que sucede en el aula.

\section{La repetición en el aula de lenguas}

En el discurso didáctico, la repetición puede observarse desde dos perspectivas, atendiendo al discurso del profesor o a los discursos de los alumnos. Se ha de tener presente que el profesor posee un rol de superioridad en la interacción frente a los alumnos. Este rol le viene dado por la institución y por su mayor competencia comunicativa o dominio lingüístico lo que le permite desarrollar una actuación discursiva mayor que la de sus alumnos y, por lo tanto, puede propiciar mayor porcentaje de repeticiones en las interacciones. También, las limitaciones lingüísticas de los alumnos en la lengua meta hace predecible que esta estrategia se presente con menor frecuencia en sus discursos que en el del profesor.

Además, durante el período de adaptación y aclimatación que se lleva a cabo entre el profesor y los alumnos los primeros días de clase para consolidarse como grupo, los participantes en estas sesiones van generando una serie de rutinas y expresiones propias del contexto académico y de la situación didáctica en las que están implicados. Estas rutinas, y las expresiones que las vehiculan van a ser similares en otros grupos con características similares. Pensemos por ejemplo en las repeticiones totales o expresiones eco tan presentes en el discurso del profesor, con la función de dar retroalimentación al alumno.

Es evidente que no es posible establecer paralelismos totales entre la conversación espontánea y el discurso didáctico; pero no es osado esperar que algunos de los objetivos que Tannen, Vigara, Briz o Herrero presentan para la repetición en la conversación puedan estar presentes en el discurso didáctico. 


\subsection{Análisis de los datos}

Los 10 profesores de español a alumnos de nivel principiante cuyos discursos han sido objeto de estudio se han servido de la repetición de diferentes formas, por lo que se ha de presentar cada una de ellas por separado. A continuación en el cuadro 1 se recogen las diferentes posibilidades de este fenómeno, en cuanto a su forma, en los discursos objeto de análisis. Esta clasificación se ha elaborado a partir del análisis de los datos y de las propuestas de Tannen (1989), Briz (1998a, 1989b) y Vigara (1995).

\begin{tabular}{l|l}
\hline \multicolumn{2}{l}{ La repetición } \\
\hline 1. Repetición total (RT) & En el mismo turno \\
\hline & En diferente turno \\
\hline 2. Repetición parcial (RP) & \\
\hline 2.1 RP de elementos & En el mismo turno \\
iniciales del enunciado & En diferente turno \\
$\begin{array}{l}\text { 2.2. RP de elementos } \\
\text { intermedios y finales del } \\
\text { enunciado }\end{array}$ & En el mismo turno \\
\hline
\end{tabular}

Cuadro 1. Diferentes tipos de repetición presentes en los discursos objeto de estudio

Como puede verse en el cuadro $\mathrm{n}^{\circ} 1$ se han presentado en los distintos discursos dos tipos de repetición (a partir de ahora R), por un lado una $\mathrm{R}$ que consiste en la reproducción exacta de la misma producción que ya ha sido emitida; y, por otro lado, una R que se caracteriza por ser una parte del enunciado la que se reproduce; por ello puede hablarse de dos tipos de R diferentes. La Repetición Total (a partir de ahora, RT) es la producción que repite literalmente un enunciado emitido por el profesor. La Repetición Parcial (a partir de ahora, RP) es la que repite sólo una parte del enunciado emitido por el profesor.

Es necesario analizar la $\mathrm{R}$ desglosada en los dos tipos presentados anteriormente, en primer lugar por su frecuencia de aparición en el discurso del profesor. Y, en segundo lugar, porque se intentará ver a través del estudio de los datos si la RP y la RT tienen una función diferente en el interior del discurso de los sujetos informantes, o si por el contrario solo las diferencia su forma.

Del mismo modo, al analizar la $\mathrm{R}$ se ha de tener en cuenta si ésta se ha emitido en el mismo turno que el enunciado que está reiterando, o si se ha realizado en un turno diferente para establecer si esa diferencia es significativa. Así, a la hora de analizar la RP también se detectan diferencias en los datos que deben ser consideradas, si la RP está repitiendo el primer elemento del 
enunciado, lo que convierte a ésta en un falso inicio; o si la RP está repitiendo otros elementos del enunciado del profesor.

\subsubsection{La repetición total}

El uso de la RT, por parte de los sujetos cuyo discurso está siendo analizado, expone al estudiante de nuevo a una producción que ya ha sido emitida, de tal forma que le ofrece la oportunidad de volver a escuchar el enunciado producido por el profesor. Al iniciar este estudio presuponíamos que esta estrategia estaba motivada por una falta de respuesta verbal o no verbal del alumno a un requerimiento del profesor, lo que indicaría que el primero no había comprendido el mensaje que intentaba transmitir el segundo; al analizar las transcripciones pudo apreciarse que esto no siempre es así.

La RT puede presentarse de dos formas. Esto responde a realidades diferentes dentro del discurso. Por un lado hemos apreciado que la $\mathrm{R}$ que se produce en el mismo turno parece motivada bien porque el profesor, por su experiencia en el aula, cree que esa emisión va a presentar problemas de comprensión a los estudiantes, sea por la forma o por el contenido; o bien porque ya mientras está produciendo el mensaje percibe de modo no verbal problemas de comprensión en sus estudiantes (Aymerich y Díaz 1992). En cambio, por otro lado, la RT que se realiza a través de diferentes turnos de los sujetos informantes está motivada por una intervención de un estudiante. Este, tras la producción del profesor, expresa de forma oral que no comprende la producción del profesor, lo que obliga al profesor a reiterar el enunciado emitido. A continuación se presentan en forma de cuadro (cuadro $n^{\circ} 2$ ) las RT producidas por los sujetos en las diferentes sesiones.

\section{La repetición total}

\begin{tabular}{l|l|l|l|l|l|l|l|l|l|l}
\hline & P1 & P2 & P3 & P4 & P5 & P6 & P7 & P8 & P9 & P10 \\
\hline Mismo turno & 4 & 1 & 4 & 5 & 1 & 8 & 0 & 2 & 5 & 5 \\
\hline Diferente turno & 0 & 2 & 1 & 1 & 4 & 5 & 0 & 4 & 4 & 2 \\
\hline
\end{tabular}

Cuadro 2. Presencia de la repetición total en los diferentes discursos

Al observar el cuadro $n^{\circ} 2$ puede apreciarse que todos los sujetos (a excepción de P7 y de P1 que sólo presenta el primer tipo de RT) presentan los dos tipos de RT, aunque con diferencias en cuanto al número de veces. La RT en el mismo turno se ha presentado en 35 ocasiones, siendo P6 el sujeto que más se ha servido de este recurso (en 8 ocasiones) y P2 el que menos (en una ocasión).

La RT tiene como objetivo dar la oportunidad al estudiante de escuchar de nuevo una producción emitida por el profesor, aunque esto no implique 
que la simple exposición a una producción en diferentes ocasiones consiga que esta sea comprendida por el estudiante, como podrá observarse.

\subsubsection{La RT en el mismo turno}

Aunque se haya dicho que la $\mathrm{R}$ puede favorecer la comprensión del alumno de un enunciado, hay ocasiones en las que esto no es así; ya que el profesor, en multitud de ocasiones, no proporciona tiempo suficiente al estudiante para que conteste. La RT en el mismo turno tiene como función principal, según Aymerich y Díaz (1992) facilitar la comprensión al estudiante o también la reparación del propio mensaje ${ }^{4}$.

De las 35 RT, solo 22 esperaban respuesta del alumno y 13 no; ya que no estaban formuladas para ser respondidas por los estudiantes, sino están motivadas por un interés del profesor en insistir sobre un mismo mensaje. De las 22 que sí esperaban respuesta, únicamente 14 repeticiones lo han conseguido, es decir menos del 50\%, en cambio 8 no han encontrado respuesta. Es significativo que menos de la mitad de las RT emitidas por el profesor hayan logrado su objetivo. Hay algunos casos en los que puede explicarse claramente cuál ha sido el problema por el que el estudiante no ha podido dar una respuesta, en cambio en otros es difícil saber si la dificultad radicaba en la forma o en el contenido de la producción emitida. O por el contrario el problema estaba en no haber facilitado al alumno el tiempo necesario para emitir una respuesta.

A continuación mostramos a través de ejemplos cómo se ha presentado ésta en los distintos discursos que han sido objeto de análisis con el fin de ejemplificar lo dicho hasta ahora.

El siguiente fragmento pertenece a la sesión del sujeto P4, está hablando con los alumnos al inicio de la sesión, es lunes y el profesor se interesa por el fin de semana de sus estudiantes.

P4 (3-4)

P: ¿qué tal el fin de semana?, ¿qué tal el fin de semana?

E: bueno

El profesor, tras la primera pregunta, no obtiene respuesta, en la segunda producción repite exactamente lo que ha dicho, no reformula la emisión. Vemos que tras la RT sí responde uno de los estudiantes. Es posible que el factor tiempo sea más importante de lo que algunos profesores piensan. Tal y como afirman algunos autores como Krashen, es importante que el alumno

4 Estas autoras las definen como modificaciones que "[...] incluyen aquellas motivadas por la autoconciencia de la dificultad de lo explicado, y su función sería la facilitación del input o bien la corrección del mensaje por el propio emisor (reparación)." (Aymerich y Díaz 1992). 
disponga del tiempo suficiente para procesar la información. Si los estudiantes pueden responder a una repetición de una pregunta, pero no a la pregunta formulada por primera vez, y si en la RT no hay ningún cambio, quizá debería tenerse en cuenta que los estudiantes necesitan más tiempo que el proporcionado para decodificar el enunciado y formular una respuesta.

En el ejemplo que mostramos a continuación de la sesión del sujeto P1, en él la RT no se da en la siguiente producción, pero sí en el mismo turno, el sujeto emite su producción, no obtiene respuesta, lo intenta con dos producciones diferentes, para finalmente volver a la primera producción.

P1 (10-15)

P: ¿qué ha pasado?, ¿eh?, ¿mucho?, ¿qué ha pasado?, intenta

E: me he perdido

El estudiante responde sin que haya habido una reformulación o sustitución de elementos léxicos en la producción del profesor. Esto lleva a pensar de nuevo que el problema es la falta de tiempo para interpretar la pregunta y elaborar una respuesta, no la complejidad del enunciado.

La siguiente producción se da durante la presentación de contenidos gramaticales, en la sesión del sujeto P9, éste está explicando a sus estudiantes la oposición de significado entre el pretérito perfecto y el pretérito indefinido. La falta de respuesta en el estudiante se debe a problemas acerca del conocimiento de la forma verbal en cuestión.

P9 (158-161)

P: ¿has estado en Lloret?, ¿has estado en Lloret?, estar es irregular

E: estuve el sábado y el domingo

Tras la producción 160, parece que si el estudiante no ha respondido a la primera producción es por el desconocimiento de la forma verbal. El profesor le ofrece la pista para que pueda responder de forma correcta. La respuesta esperada se ha producido por la ayuda proporcionada ("estar es irregular") por el profesor y no por la RT emitida. No se alcanza el objetivo de que el alumno comprenda el enunciado que el profesor emite a través de una $\mathrm{R}$ porque el problema no es de comprensión, sino que radica en la inseguridad del alumno acerca de la forma correcta del verbo que debe utilizar.

En el siguiente ejemplo, extraído de la sesión de P3, hay un problema de incomprensión de un elemento léxico presente en la producción del profesor, por lo que el estudiante no puede contestar la pregunta.

P3 (147-152)

P: um Mishi ,¿alguna vez has ido a Granada?, ¿alguna vez has ido a Granada?, (tos) ¿alguna vez has ido a Granada? 


\section{E1: ¿Granada? \\ E2: es un "city" \\ P: ¿Granada Granada conocéis Granada? \\ El: no me he ido a Granada}

P3 inicia una actividad con una pregunta un tanto desafortunada, supone un conocimiento del mundo común entre él y sus estudiantes. En este ejemplo es la intervención de otro estudiante el que resuelve un problema de contenido, no el profesor. No es la RT emitida por el profesor la que ha conseguido la respuesta del estudiante, sino la intervención de otro estudiante con la que se aclara el significado del elemento léxico desconocido. El profesor emite tres veces el mismo enunciado, lo que obliga a pensar que no ha detectado qué planteaba problemas al alumno. Si se tiene en cuenta que este fragmento ha sido extraído de la fase de Práctica Controlada y que el objetivo de enseñanza de esa sesión es la presentación de una forma de pasado, es fácil comprender la actitud del profesor: su atención se focaliza sobre el nuevo tiempo verbal, por lo que menosprecia los demás elementos de enunciado.

En el siguiente fragmento de la sesión del sujeto P8, un alumno tiene un problema de contenido al igual que el sujeto anterior.

P8 (182-185)
P: ¿y huir?, ¿huir?
E: ¿huir?
P: un policía (.) va un ladrón (.) un banco él roba el dinero ...

En estas producciones el sujeto P8 explica las irregularidades en la forma del gerundio. Este profesor pregunta a un estudiante y emite una RT, pero no obtiene la respuesta esperada (la forma correcta del gerundio). Observamos en este ejemplo cómo la reacción del estudiante es igual a la del alumno del ejemplo anterior. Ante una producción incomprensible repite la palabra, este mecanismo es el que motiva la siguiente intervención del profesor con la que intenta definir el elemento léxico en cuestión.

Otro aspecto similar en los dos fragmentos de discurso es que, antes de que el alumno pueda intervenir, el profesor ya ha repetido su enunciado; en el primer fragmento, dos veces, y en el segundo, una vez. No obstante, cuando el alumno tiene la oportunidad de intervenir con su producción informa al profesor acerca de cuál es su problema. Al igual que en los ejemplos anteriores, podría afirmarse que si el alumno dispusiera de más tiempo, quizás la RT del profesor sería innecesaria.

En los datos hay algunas RT en las que es difícil saber qué ha sido lo que ha motivado su aparición, es decir, a primera vista no se sabe si el problema 
está en la forma o en el contenido. Veamos el ejemplo siguiente perteneciente a la sesión del sujeto P9.

P9 (237-241)

P: ¿a qué hora te levantaste ayer?, ¿te levantaste?, ¿a qué hora te levantaste?

$\mathrm{E}:$ ieh?

P: me (.) es levantarse (.) no importa (.) tú tranquila, es levantarse me me levanté

Aunque en esa sesión se supone que los estudiantes conocen ya el contenido del verbo y que lo que se practica es el uso del tiempo verbal, por las producciones del sujeto P9 parece que el problema está en el contenido y no en la forma. Esta idea queda corroborada si miramos las producciones posteriores a ésta en la transcripción. En este fragmento la profesora interpreta que la alumna no reconoce el tiempo verbal empleado en la pregunta, por ello repite la pregunta. Y es ante la reacción de la alumna que la profesora cambia su percepción y reacciona comprendiendo que esta no entiende el significado del verbo. En este caso, la $\mathrm{R}$ no puede facilitar que la alumna llegue a emitir una repuesta correcta porque la profesora no ha detectado el problema.

Hemos dicho anteriormente que hay intervenciones de los profesores que contienen RT de sus producciones que no esperan respuestas del estudiante. Estas están motivadas bien por alguna reacción no verbal del estudiante o bien por una apreciación, debida a la experiencia del profesor, de la dificultad en cuanto a la forma o el contenido de la producción.

Veamos el siguiente fragmento de la sesión de Pl tras una respuesta ofrecida por un estudiante.

P1 (197-200)

P: te da igual los demás sitios, sólo quieres el lugar, sólo quieres el lugar, muy bien muy estricto

Estas producciones son el resultado de la reacción del profesor ante la respuesta del estudiante, la repetición parece que no espera nada de este último, Pl está recogiendo a modo de resumen las respuestas del estudiante a diferentes preguntas formuladas por él antes de ceder el turno a otro estudiante.

Observemos otro fragmento perteneciente a la sesión del sujeto P9, este está ejemplificando el uso de una forma verbal.

P9 (132)

P: ¿has comido paella? (.) ¿has comido paella?

Esta producción en realidad no es una pregunta que espere una respuesta sobre el contenido que se cuestiona, sino que lo que se está preguntando es el 
nombre del tiempo verbal. La profesora insiste y repite en la segunda parte de la producción la primera parte para facilitar la comprensión al estudiante.

Al principio de este apartado anunciábamos que uno de los objetivos de analizar esta estrategia es ver la relación existente entre las distintas formas que esta presenta y las funciones que cumple. A continuación presentamos un cuadro que resume la información obtenida tras el análisis realizado con el objetivo de facilitar la comparación con el resto de repeticiones que se presentan en el discurso del profesor.

Objetivos de la repetición total en el mismo turno

1. Facilitar la comprensión

1.1. SÍ se consigue si el alumno tiene la oportunidad de intervenir

1.2. NO se consigue si no se da la oportunidad al alumno de intervenir

2. Resumir información

2.1. Se supone que se consigue porque no requiere la intervención del alumno

Cuadro 3. Objetivos de la RT en el mismo turno

Como se ha podido ver a través de los ejemplos, la RT en el mismo turno que se ha producido en los discursos analizados persigue dos objetivos diferentes: facilitar la comprensión de un enunciado y resumir la información dada por los estudiantes. En el primer caso se cumple el objetivo si se permite al estudiante que intervenga, ya que de ese modo él explícitamente puede formular cuál es el problema de comprensión que plantea el enunciado producido por el profesor y facilitar que el profesor le proporcione la solución. En el segundo caso, puede afirmarse que se cumple siempre el objetivo, ya que, al no esperarse ninguna intervención del alumno, no es posible interceptar posibles problemas.

\subsubsection{La RT en diferentes turnos}

En este punto se tratan las RT emitidas a lo largo de la interacción, es decir las producciones que reiteran un enunciado emitido en turnos de palabra anteriores. Aymerich y Díaz (1992) en su estudio a estas repeticiones las llaman 'Reformulación', a nosotros no nos ha parecido conveniente este nombre ya que se puede prestar a confusiones. Preferimos hacer la distinción entre RT en el mismo turno o RT en turnos diferentes y reservarnos el término de Reformulación para la repetición que parafrasea un enunciado ya emitido.

Éstas se producen en un número menor a las anteriores; pero sorprende la alta proporción de éxito que alcanzan, ya que de las 21 ocasiones en que han apa- 
recido, 12 logran su objetivo, facilitan la comprensión de los estudiantes y obtienen una respuesta, 2 no consiguen una contestación por parte de los estudiantes y 4 no eran producciones que esperasen una respuesta oral de los estudiantes.

Recordemos ahora los datos de la RT en el mismo turno, la proporción de producciones con respuesta era mucho menor que en este caso. El porcentaje de RT en el mismo turno con éxito era menor al 50\% frente al 57,1\% de respuestas con éxito obtenido con la RT en diferentes turnos de intervención. Puede pensarse que, si las repeticiones se realizan en turnos diferentes de palabra, los alumnos disponen de más tiempo para procesar la información que si se llevan a cabo en el mismo turno, además de que las intervenciones que se producen entre el enunciado original y la RT permiten la posibilidad de añadir información a lo ya dicho; estos dos aspectos pueden ser la clave del mayor éxito de este tipo de estrategia, frente a la vista líneas más arriba.

A continuación, a través de los ejemplos, se puede apreciar cómo se han producido estas repeticiones totales en diferentes turnos de palabra. En el ejemplo que se presenta a continuación, extraído de la sesión del sujeto P2, es difícil interpretar cuál es el problema del estudiante, si la forma del enunciado, el contenido de éste o el desconocimiento de la forma verbal preguntada por el profesor. P2 (78-81)
P: vosotros
E: has leído
P: vosotros
E: habéis leído

La RT del profesor "vosotros" actúa a modo de reacción ante la respuesta dada por el estudiante, indicándole que la respuesta no es correcta y que le ofrece la oportunidad de intentarlo de nuevo. En el segundo intento el alumno sí tiene éxito.

En el siguiente fragmento se plantea un problema extraño, la RT está motivada por la incomprensión de la pregunta formulada. Parece que en este último ejemplo el problema que obliga a nuestro sujeto a formular la RT viene dado por la brevedad de las instrucciones presentes en su discurso P2 (94-99)
P: cerrar
E: cerrado
P: vosotros
E: ¿cerrar?
P: cerrar
E: habéis cerrado 
En ambos casos, P2 -ante el error en el primer caso y la falta de comprensión de las instrucciones, en el segundo- emite una RT que obtiene del estudiante una respuesta con éxito. Entre la producción en la que se emite por primera vez el elemento que causa problemas y la segunda vez que aparece éste, se encuentra otra producción que ayuda al alumno a descifrar qué es lo que se le está pidiendo. Con este tipo de preguntas tan breves el alumno que no domina la rutina de la dinámica de clase o que no está atento a su desarrollo puede tener problemas. Por esto, quizás, este tipo de repetición ha tenido más éxito que la anterior, porque permite al alumno escuchar de nuevo el enunciado tras haber intentado emitir una respuesta. El error que él ha producido y la reacción del profesor le indican cuál es la interpretación correcta, entre todas las posibles.

Presentamos a continuación un ejemplo de la sesión del sujeto P3 en el que se puede ver que la RT está motivada por un problema de incomprensión de la persona gramatical en la forma verbal.

P3 (185-190)

P: ¿qué ha hecho Cathy esta mañana?

¿qué ha hecho?

$\mathrm{E}$ : ah esta mañana hemos hecho

P: no no

¿qué ha hecho Cathy esta mañana?

E: oh se ha levantado

El sujeto P3 reacciona ante el error de la estudiante primero con una evaluación negativa, y después con una RT de la pregunta, dándole así la oportunidad a la estudiante de que repare su falta. En este fragmento puede apreciarse más claramente lo que se ha dicho en el párrafo anterior, la información que proporciona la reacción del profesor ante el enunciado del alumno, más la repetición parece que se convierten en una combinación eficaz para conseguir su objetivo.

El siguiente ejemplo está extraído de la fase de la sesión del sujeto P8, éste está dirigiendo una pregunta a un estudiante con el objetivo de practicar el presente progresivo o durativo.

P8 (210-217)

P: Barbara ¿qué estoy haciendo?

¿qué estoy haciendo?

¿qué estoy haciendo?

E: (risa) estás estás 
P: ¿sí qué estoy haciendo?

E: nada no

P: ¿no estoy haciendo nada?

E: estás pensan pensando

El profesor, al no obtener una respuesta de tipo verbal, repite su producción en dos ocasiones, la estudiante intenta contestar pero no puede, por lo que el sujeto P8 vuelve a emitir una RT; insiste, repitiendo la pregunta en dos ocasiones sin obtener respuesta. La alumna en la producción 213 intenta formular una respuesta, la profesora reacciona repitiendo su enunciado, la alumna ofrece una respuesta válida, aunque no es la que la profesora quiere, por lo que ésta reacciona incluyendo en su enunciado la respuesta de la alumna y, finalmente, ésta contesta correctamente. En este intercambio ha podido apreciarse el despliegue de estrategias llevado a cabo por la profesora para obtener una repuesta de la alumna.

En este caso, es difícil precisar qué ha hecho posible la respuesta de la alumna, si la repetición, la reformulación, o las reacciones de la profesora, pero sí que el que la alumna pudiera intervenir ha facilitado el que se produjera este complejo intercambio con final exitoso.

No todos los ejemplos muestran la complejidad del anterior, veamos el siguiente fragmento perteneciente a la sesión del sujeto P9.

P9 (229-232)
P: ¿te gustó?
E: ¿eh?
P: ¿te gustó?
E: $s i ́$

La RT del profesor está motivada en este caso por la pregunta emitida por el estudiante en su intervención en la que le está demostrando al profesor que no ha comprendido la pregunta que le ha hecho, o simplemente que no estaba atento. P9 en su segunda intervención repite textualmente lo que ha dicho en la primera y parece que esto es suficiente para el estudiante.

En el ejemplo que mostramos a continuación, extraído de la sesión del sujeto P5, se dan los dos tipos de RT, en el mismo turno y en turnos diferentes.

P5 (196-199)

P: ¿a qué hora has (.) llegado a la escuela?

¿a qué hora has (.) llegado a la escuela?

E: eh

P: ¿a qué hora (.) has llegado a la escuela? 
Este sujeto en un mismo turno repite su primer enunciado; la reacción del estudiante le indica que éste tiene problemas para responder, por lo que vuelve a repetir la producción. En este intercambio, el alumno desconoce el tiempo de la forma verbal empleado por la profesora lo que le impide contestar. Si continuamos mirando la transcripción, veremos que con estas dos RT el profesor tampoco consigue su objetivo. Este necesita ir descomponiendo el enunciado en unidades menores, clarificándole al estudiante los diferentes elementos que lo componen para que así pueda elaborar una respuesta.

Parece, pues, que la RT que se produce en turnos diferentes al enunciado original está motivada, principalmente, por una pregunta del estudiante en la que éste le hace saber al profesor que existe un problema, ya sea de desconocimiento de algún elemento léxico, o de falta de atención. Hemos visto cómo este tipo de RT tiene más éxito que la anterior. Este podría ser debido a tres factores:

a. El estudiante es el que pide la RT, por lo que su nivel de concentración ante la repetición es mayor, es más si éste está preparado para recibir una repetición el tiempo del que dispone para decodificar el enunciado y elaborar el suyo también es mayor.

b. El problema del estudiante puede ser simplemente que no ha podido oír a la profesora y sí conoce la respuesta a la pregunta por lo que tras la RT él puede contestar de forma correcta.

c. Las intervenciones intermedias que emiten la profesora o el estudiante añaden información al enunciado original lo que también redunda en la facilitación de la comprensión

A continuación presentamos, a modo de resumen, un esquema que recoge la información que se ha presentado en este apartado:

Objetivos de la repetición total en diferente turno

1. Facilitar la comprensión ante una duda planteada por un alumno por desconocimiento de algún elemento léxico

1.1. Sí cumple su objetivo porque es el alumno quien la pide

2. Facilitar la comprensión ante una duda planteada por un alumno por falta de atención

2.1. SÍ cumple su objetivo porque es el alumno quien la pide

Cuadro 4. RT en diferente turno 
En este apartado ha podido apreciarse, a través de los datos presentados, que la repetición que se produce en un turno de habla diferente al del enunciado original cumple sus objetivos en un porcentaje mayor que la vista en el apartado anterior. Esto es debido a que este tipo de RT es solicitada por el alumno, no se produce, como la anterior, por una percepción del profesor que le indica que quizá sea necesario repetir el enunciado. Por ello podría afirmarse que las percepciones del profesor de lo que es necesario que sea repetido no siempre se ajustan a la realidad.

\subsubsection{Repetición Parcial}

Se han agrupado bajo esta etiqueta las producciones del profesor que repiten de forma parcial otros enunciados emitidos por él mismo; es decir, los enunciados que están formados por fragmentos de su propio discurso.

La Repetición Parcial (a partir de ahora RP) es una de las modificaciones que en mayor número hemos encontrado en los discursos de los sujetos registrados. 137 producciones contienen alguno de los dos tipos en los que se ha dividido esta categoría. La RP está presente en los discursos de todos los sujetos; el profesor que más se ha servido de este recurso ha sido P9 con 21 producciones; el que menos lo ha utilizado ha sido P8, con 6 producciones; los demás sujetos se mueven entre estos dos valores.

Las RP, aparentemente, se presentan en todos los sujetos bajo una forma similar, ya que se trata de repeticiones. Pero analizándolas de forma exhaustiva pueden encontrarse diferencias en la forma de realizarse. Hemos observado RP de dos tipos diferentes: las que repiten elementos léxicos presentes en el inicio de la producción original de nuestros sujetos, a modo de reparaciones o de Falsos Inicios (Van Lier, 1988). Y por otro lado, las RP que repiten elementos que aparecen en el interior o en el final de la producción original.

\subsubsection{Repetición Parcial (falsos inicios)}

Los sujetos cuyo discurso está analizándose han utilizado este recurso en 31 ocasiones, es decir, han iniciado, ese número de veces, una producción y sin llegar a terminarla la han reiniciado, dando lugar así a una producción que contiene en sí misma un doble inicio. Pocas veces se da el falso inicio en una segunda producción, sino que el falso inicio, si no hay una pausa lo suficientemente larga como para ser considerada de forma contraria, se da en la misma producción. Veamos a continuación con qué frecuencia ha aparecido este fenómeno en las diferentes sesiones. 


\begin{tabular}{c|c}
\hline \multicolumn{2}{|c}{ Repetición parcial: falsos inicios } \\
\hline P1 & 2 \\
\hline P2 & 3 \\
\hline P3 & 5 \\
\hline P4 & 4 \\
\hline P5 & 5 \\
\hline P6 & 3 \\
\hline P7 & 3 \\
\hline P8 & 2 \\
\hline P9 & 2 \\
\hline P10 & 2 \\
\hline
\end{tabular}

Cuadro 5. Presencia de la Repetición Parcial en el inicio del enunciado

Como puede observarse en el cuadro $\mathrm{n}^{\circ} 5$, este fenómeno está presente en el discurso de todos los sujetos, aunque no es un fenómeno que se haya dado con mucha frecuencia.

La RP que se produce en el inicio de la producción en los discursos de los informantes se origina por las percepciones del profesor sobre su discurso o sobre la atención que le están prestando sus interlocutores. No es necesario para que esta aparezca que los alumnos le pidan de forma verbal que repita parte del enunciado, sino que puede ser originada también por las reacciones no verbales de los alumnos. Veamos a continuación a qué responden las RP encontradas en los discursos de los sujetos informantes.

\section{Objetivos de la repetición parcial en el inicio del enunciado}

1. Reparar un enunciado ambiguo o incorrecto

2. Manifestar duda sobre su propio discurso

3. Llamar la atención a los interlocutores

4. Insistir sobre un aspecto concreto

Cuadro 6. Objetivos que cumple la Repetición Parcial en el inicio del enunciado

A la vista del cuadro anterior pueden apreciarse los cuatro objetivos diferentes a los que responde la presencia de la RP en el inicio del enunciado. En primer lugar se encuentran las RP que responden al objetivo de reparar una producción del profesor poco clara, ambigua o incorrecta. En segundo lugar, 
están las RP que manifiestan dudas o vacilaciones del sujeto al formular una producción. En tercer lugar, las que tratan de hacer una llamada de atención a los interlocutores sobre el discurso que se está emitiendo. En último lugar tenemos las que están motivadas por la necesidad del profesor de insistir sobre un aspecto concreto de su enunciado.

Vamos a detenernos en algunos de los ejemplos de RP para ver a través de éstas cómo los sujetos formulan sus objetivos.

El primer ejemplo es un extracto de la sesión del sujeto P10 que se produce como respuesta a un problema de vocabulario, al no ser comprendido un elemento léxico por un alumno, pero, si nos fijamos la RP, se da en el principio del enunciado antes de que aparezca el término que ha causado el problema. P10 (106)

P: se puede (.) se puede (.) admitir (.) en inglés póster (.) pero en español hay otra palabra

En este caso puede decirse que el profesor, al iniciar su producción, tiene un momento de duda o vacilación. Reinicia su producción con una RP para, seguidamente, terminarla. Es interesante fijarse en cómo el falso inicio va enmarcado entre dos pausas, esto nos sirve para corroborar la idea anunciada anteriormente de que esta RP responde a una duda al iniciar la producción.

En el ejemplo del sujeto P5 que se muestra seguidamente puede verse un caso similar al del discurso del sujeto P10. La profesora, a través de la interacción, está practicando un nuevo tiempo verbal.

P5 (227)

P: ¿a quién (.) a quién has visto (.) a quién de las dos has visto primera esta mañana?

Esta producción responde a un intento de reparación de una pregunta mal formulada. La parte del enunciado que se repite está enmarcada entre dos pausas (como en el discurso de P10) lo que puede ser significativo. Ya que igual que se ha visto antes parece que cuando el profesor duda ante los elementos léxicos o la estructura seleccionados lo manifiesta a través de los silencios que limitan la parte repetida.

El siguiente ejemplo, extraído de la sesión del sujeto P3, muestra una situación similar a la anterior, se da tras la introducción del mismo tiempo verbal. P3 (154-155)

\section{P: ¿es correcto nunca?} ¿es correcto nunca me he ido?

Como en los ejemplos anteriores, puede afirmarse que esta RP responde a una reparación en la pregunta hecha inicialmente, la profesora está corrigiendo 
a un estudiante, lo incorrecto en la producción de este no es "nunca" es "me". Tras la primera producción, la profesora se percata de que no queda claro qué es lo incorrecto, porque no aparece el elemento sobre el que se está discutiendo, por ello es por lo que es necesario reparar el enunciado, para incluir el elemento sobre el que duda.

En el siguiente ejemplo perteneciente a la sesión del sujeto P8, éste tiene una actuación similar al sujeto P3, en su RP también hay una reparación de lo dicho en la primera producción.

P8 (186-187)

P: $y$ él ve a la policía, ve al policía (.) y se queda en el banco

Aunque en este caso la corrección que hace el sujeto no afecta al contenido informativo transmitido a los estudiantes (en ese nivel los estudiantes no conocen la diferencia entre flexión de género y significado, y es difícil saber si la pueden percibir), sí que la RP responde a la necesidad de reparar lo dicho o de formular producciones correctas sin ambigüedades. La RP está motivada por el intento de reparar las incorrecciones de su propio discurso.

Mostramos a continuación un ejemplo de la sesión del sujeto P7 que responde a objetivos distintos.

P7 (239)

P: con el verbo estar (.) con el verbo estar cuando nos referimos a un lugar

El sujeto P7, con la RP, parece insistir sobre un punto concreto de su producción, es un intento de focalizar la atención sobre el contenido de lo que está explicando.

Otro caso diferente es el del ejemplo extraído de la sesión del sujeto P4, la llamada de atención que realiza el profesor no es sobre el contenido que se está presentando, sino que es para avisar de que se va a producir un cambio de actividad en ese momento.

P4 (339-341)

\section{P: vamos a}

vamos a escuchar (.) vamos a escuchar

vamos a escuchar un caset en el que hablan dos personas

En estas producciones, el sujeto $\mathrm{P} 4$ anuncia una nueva actividad en el desarrollo de la sesión. La presentación se hace en tres producciones, hasta la última no aclara qué se realizará a continuación.

La RP que se propone llamar la atención del interlocutor cumple su función al aislar el contenido informativo entre pausas, obligando al alumno a 
centrar su atención en el elemento repetido, de esa forma reconduce la atención del estudiante hacia la parte del enunciado seleccionada.

Tras los ejemplos presentados, parece que estas RP responden a dudas o vacilaciones de nuestros sujetos. Estos, tras iniciar su enunciado, perciben que con sus palabras no queda claro lo que se está emitiendo. En la RP hay una reestructuración o reorganización del contenido o de los elementos léxicos ya emitidos. La función de esta RP se debe, por un lado, a la creencia o la intuición por parte del profesor de que no es comprendido por los alumnos; y, por otro lado, también está motivada por el interés en insistir sobre algún contenido nuevo, para que quede claro lo que se está introduciendo o preguntando. Por último, también se ha visto que la RP puede estar motivada por el interés del profesor en reparar alguna parte de su enunciado considerada incorrecta.

A continuación presentamos un esquema que recoge la información expuesta en este apartado.

\begin{tabular}{l|l|l}
\hline \multicolumn{2}{l}{ Repetición parcial: Falsos inicios } \\
\hline \multicolumn{2}{c}{ Motivada por la percepción del profesor de que su producción } \\
\hline 1. no es comprendida & l. facilitar la comprensión \\
2. es incorrecta & Con el objetivo de & 2. reparar el error \\
3. es ambigua & 3. deshacer la ambigüedad \\
4. es importante & 4. llamar la atención \\
\hline
\end{tabular}

Cuadro 7. Repetición parcial: falsos indicios

\subsubsection{Repetición Parcial de otros elementos del enunciado}

Bajo la etiqueta de RP de otros elementos del enunciado como los intermedios y finales de una producción, se han agrupado todas aquellas emisiones que repiten elementos léxicos de una producción o parte de su estructura y que no se encuentran en el inicio de la misma, es decir las que no corresponden a los falsos inicios.

Estas RP son muy numerosas en los discursos que están analizándose, se han presentado en 106 producciones. A continuación presentamos una tabla que recoge exactamente la cantidad de veces que ha aparecido este recurso en los distintos discursos que están analizándose. En esta tabla puede apreciarse que la RP de otros elementos del enunciado que no se corresponden con el inicio de este se ha presentado de forma desigual. 


\begin{tabular}{c|c}
\hline \multicolumn{2}{c}{ Repetición parcial: otros elementos } \\
\hline P1 & 15 \\
\hline P2 & 8 \\
\hline P3 & 10 \\
\hline P4 & 6 \\
\hline P5 & 6 \\
\hline P6 & 14 \\
\hline P7 & 10 \\
\hline P8 & 3 \\
\hline P9 & 19 \\
\hline P10 & 16 \\
\hline
\end{tabular}

Cuadro 8. Repetición parcial de otros elementos

Hay diferencias en estas repeticiones teniendo en cuenta si se producen por una motivación proveniente del profesor o si, por el contrario, están motivadas por una intervención del alumno. Esta diferencia es importante, pues a diferente origen en la motivación de la RP le corresponde una forma de realizarse diferente. Las RP que se producen como reacción a una intervención del alumno, se dan en un turno diferente al de la producción original. En cambio las RP que no responden a una intervención del alumno se dan, la mayoría, en el mismo turno que la producción original.

Tras lo expuesto en el párrafo anterior parece clara la necesidad de desglosar este tipo de repetición en dos grandes apartados: las que se producen en el mismo turno y las que se producen en turnos diferentes al enunciado al que repiten.

4.1.2.3. Repetición Parcial: repetición de otros elementos del enunciado en el mismo turno

En este apartado se presenta el análisis de las producciones que contienen una RP de algún elemento del enunciado original que se han emitido en el mismo turno que éste. Los enunciados que incluyen este tipo de RP suman un total de 113 producciones de las cuales la gran mayoría, 96, se han realizado en el mismo turno. Esto significa que el $84,9 \%$ de las RP las ha emitido el profesor sin que mediara una intervención oral del estudiante.

El cuadro que se presenta a continuación reúne los diferentes objetivos perseguidos con el uso de este recurso por los sujetos cuyo discurso está siendo analizado. 


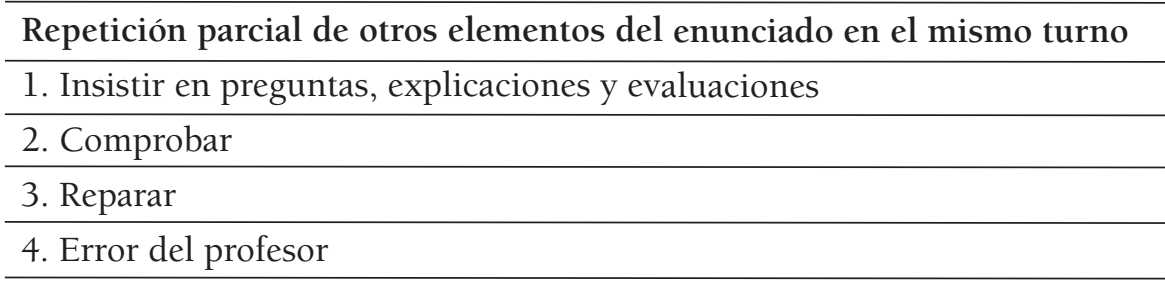

Cuadro 9. Objetivos de las repeticiones parciales de otros elementos del enunciado en el mismo turno

En primer lugar, se presentan las que responden al interés de insistir en una pregunta, explicación o evaluación; es decir marcar de forma explícita algún elemento léxico que normalmente es el objeto de estudio en esa sesión. Este tipo de RP que responde al interés de insistir sobre algún contenido se ha dado tanto en preguntas hechas a los estudiantes, como en las explicaciones de los contenidos en el discurso de los profesores. También se han realizado repeticiones de este tipo en la reacción evaluadora del profesor a las respuestas emitidas por los estudiantes y en las instrucciones para realizar alguna actividad.

A continuación mostramos algunos fragmentos de las sesiones analizadas para ver cómo se han presentado estas RP en algunos de los discursos de los sujetos.

El siguiente ejemplo, de la sesión del sujeto P6 en el que este sujeto utiliza la RP para hacer hincapié en las correcciones hechas a las respuestas incorrectas de los alumnos.

P6 (145-146)

E: Ana necesita tomar la basura

P: necesita (.) tirar (.) tirar

El sujeto P6 sólo repite el elemento que acompaña a la incorrección presente en el enunciado del estudiante, no reproduce toda la producción del estudiante.

Veamos a continuación un fragmento de la sesión del sujeto P1.

P1 (106-107)

P: pero (.) una lectura (.) rápida (.) rápida eh no hace falta que mires en el diccionario sólo una lectura rápida (.) para una idea de cómo es la academia

Vemos la insistencia que muestra este sujeto en que realmente los estudiantes hagan una lectura "rápida" del texto. Ya que éste, por su experiencia en el aula, sabe que en actividades de lectura los alumnos lo primero que hacen ante un texto es coger el diccionario e iniciar la búsqueda de todas las palabras desconocidas. Y, en este caso, no es ese su objetivo. 
Otro ejemplo similar lo vemos en la sesión de P2. También se repite el elemento final del enunciado.

P2 (265)

P: hacemos dos grupos para hacer un juego un juego

En este enunciado parece que la repetición está motivada por cuestiones diferentes. Hay un cambio de actividad y la profesora quiere dejar claro el tipo de tarea que va a iniciarse a continuación.

En segundo lugar, vamos a ver las RP que tienen el objetivo de comprobar que los estudiantes comprenden lo que se está diciendo en la producción que le precede. En este caso, las repeticiones aparecen en otra producción diferente.

El ejemplo que presentamos está extraído de la sesión del sujeto P5. P5 (39-41)

P: y decíamos que cuando hablábamos de todos los días ¿qué más?

todos los días

P5 insiste en los marcadores temporales que se usan con el presente de habitualidad. Si se mira la transcripción, las producciones comprendidas entre la 61 y la 63 repiten el mismo patrón discursivo.

P5 (61-63)

P: y cuándo decíamos la semana que viene ¿qué más?

ah la semana que viene

También en el discurso del mismo sujeto encontramos otras producciones similares. La profesora va repitiendo las partes del enunciado que aportan los elementos léxicos que quiere que recuerden los estudiantes. Por ello no se producen repeticiones totales, quiere que ellos focalicen su atención en aspectos concretos de sus enunciados: las partes que se repiten.

El siguiente ejemplo pertenece a la sesión del sujeto P9.

P9 (250-251)

P: ¿sabéis lo que es un curriculum? ¿curriculum?

El profesor emite su pregunta, intuye que no es comprendido, por lo que a continuación repite el elemento cuestionado en la pregunta. El objetivo de estas producciones es comprobar si es necesario explicar el contenido de ese elemento léxico. 
En tercer lugar presentamos las RP que funcionalmente son reparaciones que el profesor hace de su propio discurso. Es un mecanismo discursivo por el cual el profesor se autocorrige.

El siguiente ejemplo es de la sesión del sujeto P8.

P8 (23)

P: entonces por el verbo (.) ya se sabe qué persona de qué persona hablamos

Esta producción pertenece a una respuesta dada por el profesor a un estudiante, el sujeto P8 emite una estructura incorrecta (omite la preposición) y se da cuenta de ello tal y como puede apreciarse en la segunda parte del enunciado en la que repara su error.

El siguiente ejemplo pertenece a la sesión del sujeto P6.

P6 (259-260)

P: entonces (.) Sari quieres (.) empezar tú a leer quieres empezar a leer

En la segunda producción, el sujeto P6 elimina el pronombre personal sujeto, que estaba presente en la primera producción, y el nombre de la estudiante que en la primera producción ha usado. Parece haberse dado cuenta de que en realidad es redundante el uso del pronombre personal, más el nombre de la alumna, cuando el verbo ya indica la persona gramatical y el espacio del aula no es tan grande como para no poder interpretar a qué persona se dirige el profesor en cada momento.

Por último, hay que decir que también se han presentado RP que no responden a ningún objetivo aparente del profesor, más bien al contrario. Podrían considerarse lapsus, o errores no detectados por los sujetos y que por lo tanto no han podido ser objeto de autocorrección. Veamos un ejemplo.

P3 (102-103)

P: los participios de los verbos terminados en ar y de los verbos (.) terminados en ar le añadimos ado y los verbos terminados en er ir añadimos ido

En este fragmento no puede interpretarse a qué es debida esta RP. El enunciado que emite el profesor es de difícil comprensión, la primera parte parece no estar clara, pero con la RP del enunciado tampoco se deshace la ambigüedad; más bien podría afirmarse que se ha producido un error que no ha sido detectado por el sujeto.

4.1.2.4. Repetición Parcial: repetición de otros elementos del enunciado en diferente turno

En este apartado se analizan las RP emitidas por el profesor que están motivadas por la intervención de un estudiante del grupo. Este tipo de RP se ha 
presentado en un número mucho menor que las anteriores, sólo hemos encontrado 20, que representan un $17,7 \%$. Tenemos que decir que este tipo de RP no aparece en el discurso de todos los sujetos informantes, por ejemplo en el discurso de la sesión del sujeto P8 no hay ninguna producción que contenga este tipo de modificación. Además, esta RP aparece en el resto de los sujetos con una frecuencia mínima. En los sujetos en los que está presente en mayor número es en P1 y P7 (5 veces), a continuación, le siguen P2, P4 y P10 (2 veces) y en los demás sujetos 1 vez.

Este tipo de RP se ha presentado mayoritariamente como consecuencia de un requerimiento hecho por el alumno al profesor, es decir, con el objetivo de responder a un alumno; también se han encontrado estas repeticiones con otros objetivos diferentes. A continuación mostramos en un cuadro a qué es debido la presencia de este tipo de RP.

\section{Repeticiones de otros elementos en diferente turno}

1. Responder a un requerimiento de un alumno

2. Otras razones

Cuadro 10. Objetivos de las repeticiones parciales de elementos intermedios y finales en diferente turno

Como puede apreciarse a la vista del cuadro $\mathrm{n}^{\circ} 10$, es difícil establecer cuáles son los objetivos del profesor para emitir en su discurso estructuras de este tipo. Hay fragmentos de discurso en los que puede apreciarse de forma clara que la presencia de la RP está motivada por la intervención de un estudiante, pero en otros casos no ha sido así. A continuación se mostrarán algunos ejemplos para analizar el porqué de su presencia en el discurso de distintos profesores.

El primer ejemplo que presentamos está motivado por una intervención del estudiante como consecuencia de no haber oído la pregunta de la profesora. Estas producciones se emiten en la sesión del sujeto P2.

P2 (198-200)

P: ¿has ido a Argentina alguna vez Kiyokatsu?

E: más alto

P: ¿has ido a Argentina alguna vez?

El estudiante, tras la RP de la profesora, puede contestar, ya que el único problema que había para no hacerlo antes era no haber podido escuchar bien la pregunta.

Veamos a continuación otro ejemplo. En el fragmento de la sesión del sujeto P3 parece que una estudiante tiene problemas para contestar la pregunta hecha por el profesor. 
P3 (196-198)

P: Ingrid ¿qué has hecho esta mañana?

E: um

P: esta mañana

La intervención de la profesora que contiene la RP parece que intenta ayudar a la estudiante para que formule una respuesta adecuada. En este caso no podemos decir que la estudiante no ha escuchado la pregunta, sino más bien que desconoce cómo formular la respuesta. La profesora en la segunda producción omite la forma verbal -que podría ser el origen del problema por ser un verbo irregular-, y repite la marca temporal que acompaña al tiempo que es el objetivo de enseñanza de esa sesión.

El ejemplo que presentamos a continuación está extraído de la sesión del sujeto P7. En este fragmento es interesante observar cómo, a causa de un problema de significado, la RP se da 13 producciones más tarde que la producción original.

P7 (103- 116)

P: tenéis que leer (.) um (.) es un diario sabéis que es un diario

$\ldots \ldots$

P: tenéis que leerlo y contestar a las preguntas

En el primer turno de habla que se presenta, la profesora ya emite una repetición del último sintagma de su producción para que quede claro cuál es el objetivo de la actividad que presenta; pero, además, entre la primera producción que contiene la instrucción dada por la profesora y su RP hay toda una serie de producciones en las que el sujeto P7 interactúa con los estudiantes para asegurarse de que comprenden los elementos léxicos de la instrucción. Esta RP responde a la necesidad del sujeto de retomar el hilo de las instrucciones dadas al inicio de la fase, no parece que el problema sea la falta de comprensión del enunciado. Así, de esta forma, consigue retomar el objetivo de su primera producción: presentar una actividad (diferentes autores como Halliday y Hassan, 1967; Martinell, 1974, entre otros, analizan la repetición como un mecanismo de conexión).

El ejemplo que se presenta a continuación, perteneciente a la sesión del sujeto P10, es un caso mixto -al igual que el anterior-, ya que en el encontramos RP en diferente turno y RP en el mismo turno.

P10 (304-306)

P: normalmente las acabadas en consonante o en o son masculinas (.) no todas 
E: pero (.) has dicho el mapa

P: he dicho (.) no todas (.) la mayoría (.) pero no todas

La RP está motivada por la reacción de un estudiante a una explicación de P10. El profesor en la segunda producción emite una RP de la primera producción y que, a su vez, también contiene una RP.

Puede afirmarse, tras los ejemplos vistos, que la RP de elementos intermedios o finales de un enunciado puede aparecer por diversos motivos. El profesor puede querer insistir en algún elemento de los considerados como objetivo de enseñanza, o insistir en elementos presentes en las instrucciones; también, por su experiencia puede considerar que hay elementos que pueden plantear dificultad y que es necesario que sean repetidos.

A continuación presentamos un cuadro, a modo de resumen, que recoge la información más relevante acerca de la RP de otros elementos, ya sea en el mismo turno o en diferente turno.

Objetivos de la repetición parcial de otros elementos

1. En el mismo turno con el objetivo de insistir, comprobar, reparar, error o lapsus.

1.1. su éxito es menor porque no siempre permite intervenir al alumno

2. En diferente turno con el objetivo de responder o ayudar al alumno

2.1. Su éxito es mayor porque la ha solicitado el alumno

3. En diferente turno con el objetivo de cohesionar el discurso

Cuadro 11. Objetivos de la RP de otros elementos

Como ha podido apreciarse, la RP de otros elementos del enunciado original también presenta mayor índice de éxito si su presencia está motivada por un requerimiento del alumno.

\section{Conclusiones}

Si el objetivo de este trabajo es llegar a establecer las funciones de la repetición en el discurso didáctico y ver los posibles paralelismos con su función en la conversación, una vez analizadas sus formas y funciones en el discurso didáctico es necesario, pues, recordar lo expuesto en el apartado 2 de este mismo trabajo sobre la repetición en la conversación (Tannen, 1989; Briz, 1998a y 1989b; Vigara, 1995; y Herrero, 1995).

Las funciones que esta estrategia discursiva en la lengua conversacional cumple son básicamente la de permitir emitir producciones que cumplan la función de evitar los silencios, la de facilitar la comprensión de los enunciados 
al interlocutor, la de conectar los diferentes enunciados entre sí, la de facilitar la interacción y la de dotar de coherencia a la conversación a través de la implicación interpersonal.

Tras el análisis de los datos que poseemos puede afirmarse que la repetición en los discursos que han sido objeto de estudio cumple las siguientes funciones: facilitar la comprensión, resumir la información presentada previamente, reparar enunciados ambiguos o incorrectos, manifestar dudas, llamar la atención de los interlocutores, necesidad de insistir en algún aspecto concreto, responder a los requerimientos de un alumno y ayudar a un alumno.

Al comparar las funciones de la repetición en conversaciones espontáneas y en el discurso didáctico, se aprecia que hay una función que coincide en ambos actos: facilitar la comprensión. Para cumplir esta función discursiva el profesor se puede servir de diferentes estrategias: resumir la información, reparar enunciados que percibe como ambiguos o incorrectos, manifestar duda o insistir en algún aspecto concreto.

Sin embargo, casi no se han encontrado repeticiones con otras funciones. Así, no nos ha sido posible encontrar en los datos manejados ejemplos que puedan ejemplificar la presencia de repeticiones con el objetivo de dotar de coherencia a la conversación a través de la implicación interpersonal. Y tan solo se ha encontrado un caso de repetición con el objetivo de conectar los diferentes enunciados entre sí.

Es posible que en otras situaciones de enseñanza en las que se establezcan otras dinámicas discursivas diferentes y en las que el profesor y los alumnos tengan roles más igualitarios esas otras funciones también pudieran aparecer. Es decir, se han de analizar situaciones de enseñanza en las que prime una idea de comunicación entendida como la base que ha de permitir la construcción del conocimiento, primando esta la fluidez por encima de la precisión (Thornbury \& Slade, 2006). Es por lo tanto necesario seguir trabajando con corpus más amplios y de situaciones de enseñanza-aprendizaje más diversas con el fin de ver si los resultados a los que se ha llegado con este estudio pueden ser extrapolables a otros discursos didácticos o si, por el contrario, son específicos de las situaciones analizadas.

\section{Referencias bibliográficas}

Allwright, D. (1988): Observation in the Language Classroom, Applied Linguistics and Language Study. Londres, Longman.

Aymerich, M. y Díaz, L. (1992): La repetición en el discurso del profesor de español como lengua extranjera. Memoria de Master inédita, Universitat de Barcelona.

Briz, A. (1998a): El español coloquial en la conversación. Esbozo de pragmagramática. Barcelona, Ariel Lingüística. 
Briz, A. (1998b): El español coloquial: Situación y uso. Madrid, Arco/Libros

Chaudron, C. (1983): "Foreigner Talk in the Classroom- An Aid to Learning?". En Seliger y Long (eds.), Classroom Oriented Research in Second Language Acquisition. Cambridge, Newbury House Publishers, págs. 285-300.

Ferguson, C.A. (1977): "Baby talk as a simplified register". En Snow, C.E. y C.A. Ferguson (eds.) (1977), Talking to children. Language input and acquisition. Londres, CUP., págs. 209-235.

Hatch, E. (1992): Discourse and Language Education. New York, CUP.

Herrero, G. (1994): "Las construcciones eco: exclamativas-eco en español". En Actas del I Simposio sobre Análisis del Discurso Oral. Almería, Universidad de Almería, págs. 125-145.

Lightbown, P. \& Spada, N. (1993): How Languages are Learned. Oxford, OUP.

Martinell, E. (1974): El encadenamiento por repetición en la estructura coloquial. Tesis doctoral inédita, Universidad de Barcelona.

Mercer, N. (2010): "The effective use of talk in the classroom". En Interacció comunicativa i ensenyament de llengües. València, Universitat de València.

Salaberri, $M^{a}$ S. (1988): "Forma y función de la repetición en el discurso del profesor en clases de inglés como lengua extranjera”. En Vázquez, I. e I. Guillén (eds.) (1988), Perspectivas pragmáticas en lingüística aplicada. Zaragoza, Ediciones ANUBAR, págs. 325-330.

Stern, H.H. (1983/1987): Fundamental Concepts of Language Teaching. Oxford, OUP.

Tannen, D. (1989): Talking Voices. Repetition, dialogue and imagery in conversational discourse. Cambridge, CUP.

Thornbury, S., y Slade, D. (2006): Conversation: From description to pedagogy. Cambridge, CUP.

Vigara, A. M.. (1992): Morfosintaxis del español coloquial. Esbozo estilístico. Madrid, Gredos.

Vigara, A. M. (1995): "Comodidad y recurrencia en la organización del discurso coloquial". En Actas del I Simposio sobre análisis del discurso oral. Almería, Universidad de Almería, págs. 175-208. 